\title{
Aetiology of infertility among females seeking treatment at a tertiary care hospital in Sri Lanka
}

\author{
T S Palihawadana ${ }^{1}$, P S Wijesinghe ${ }^{1}$, H R Seneviratne ${ }^{2}$ \\ (Index words: infertility, aetiology, ovulatory dysfunction, sexual dysfunction)
}

\begin{abstract}
Objectives Aim of this study was to describe the proportion of contributory factors of female infertility in a population that sought fertility treatment. Furthermore, the clinical findings and underlying pathologies associated with ovulatory dysfunction were also sought.

Methods A cross-sectional study was carried out at the infertility clinic of the North Colombo Teaching Hospital. New clinic attendees were recruited and both partners had a detailed clinical interview. The women underwent a baseline pelvic ultrasound scan, assessment of ovulation and a hormone profile. Tests for tubal patency were carried out when clinically indicated.

Results Ovulatory dysfunction was noted in $53 \%(n=218)$. Clinical and investigatory findings associated with ovulatory dysfunction included irregular menstrual cycles, acanthosis nigricans, hirsutism, polycystic ovary syndrome, a LH:FSH ratio of $>1$, and increased TSH or testosterone levels. Unilateral tubal occlusion was seen in $9.1 \%(n=18)$ while it was bilateral in $1 \%(n=2)$. Abnormalities in sexual function were noted in $10.8 \%$ $(n=56)$.

Conclusions Abnormalities in ovulation were common. Clinical findings that could be used to recognise women at risk of ovulatory dysfunction were identified. Abnormalities in sexual function, which are often overlooked in the clinical management of infertility, were seen in over $10 \%$ of patients. Tubal factor infertility is rare.
\end{abstract}

Ceylon Medical Journal 2012; 57: 79-83

\section{Introduction}

Infertility is defined as a couple's inability to conceive after a period of twelve months of regular unprotected intercourse [1]. One in six couples experience a delay in conception while only a proportion of them would seek medical treatment [2]. Infertility could result from a wide spectrum of abnormalities found in either partner or sometimes in both. Though the incidence does not seem to differ significantly in different populations the aetiology of infertility has shown wide variations [1].
An understanding of the pattern of underlying pathologies would be useful for the clinicians to decide on suitable investigations and formulate management plans. Not many studies have been conducted in Sri Lanka to describe the aetiology of infertility.

Ovulatory dysfunction is the most common cause of female factor infertility whereas other causes include fallopian tube occlusion, congenital or acquired abnormalities of the genital tract and pathologies of the pelvic cavity $[1,3,4,5]$. In nearly $20-25 \%$ of couples no abnormality could be detected after investigations and are termed as having "unexplained infertility" [6].

Ovulatory dysfunction is a common finding among women with infertility [7]. Abnormalities in ovulation are broadly divided into three categories according to the gonadotropic status of the individual. These categories are important in deciding the treatment as well as in counselling. The WHO group I named hypogonadotropic hypogonadism includes women with reduced activity of the hypothalamus and the pituitary resulting in under-stimulation of the ovaries. The WHO group II named normogonadotropic hypogonadism consists of women with abnormal ovarian activity in spite of normal gonadotropins. Polycystic ovary syndrome is the main contributor of this group while other endocrinopathies can also lead to ovulatory dysfunction. WHO group III or hypergonadotropic hypogonadism is made up of women with ovarian failure in whom the lack of ovarian response results in the loss of negative feedback and therefore a rise in gonadotropin levels [8].

This study was aimed at describing the proportion of common aetiological factors among females who sought treatment for infertility. It also attempted to assess the association between clinical findings and ovulatory dysfunction in this population.

\section{Methods}

The study was carried out at the Infertility Clinic of the North Colombo Teaching Hospital, Ragama, Sri Lanka.

\footnotetext{
${ }^{1}$ Department of Obstetrics and Gynaecology, Faculty of Medicine, University of Kelaniya and ${ }^{2}$ Department of Obstetrics and Gynaecology, Faculty of Medicine, University of Colombo, Sri Lanka.
}

Correspondence: PSW, e-mail: <prasanthaw@gmail.com>. Received 12 January and revised version accepted 23 April 2012. Competing interests: none declared. 
A cross-sectional study design was employed and the participants were followed up till the investigatory work up was completed. Participants were recruited at the initial presentation to the clinic after written consent and were assessed with a detailed clinical interview and a clinical examination to identify risk factors for infertility. The sexual function was also assessed at the clinical interview.

The female participants had assessment of ovulation, an endocrinological assessment and assessment of anatomical abnormalities in the genital tract. Ovulation was assessed by ultrasound follicle tracking with a followup method which is described in outcome variables. The endocrinological assessment included FSH, LH, prolactin, $\mathrm{TSH}$ and testosterone levels done on the 2nd day of the menstrual cycle.

A baseline pelvic ultrasound scan was undertaken on the second day of the menstrual cycle in all women to evaluate the uterus and to define the ovarian morphology. The tubal patency was evaluated only if clinically indicated and diagnostic laparoscopy with chromotubation was carried out in the presence of clinical features suggestive of pelvic pathology such as pelvic pain or dysmenorrhoea as recommended in NICE Guidelines [9]. In the absence of such clinical features, hysterosalpingography (HSG) was undertaken.

All clinic attendees were assessed for suitability for the study. Those who were either on any medication to enhance fertility or those who had treatment within the preceding six months were excluded.

Ovulation was assessed according to the regularity of the menstrual cycle. In women with regular menstrual cycles, if ovulation was confirmed in the first cycle they were categorised as having regular ovulation. In those where ovulation was not detected in the first cycle, a follow up was done for three consecutive cycles with follicle tracking. Those with ovulation in some of these cycles were termed as having inconsistent ovulation while if ovulation was detected in none of the cycles they were termed as having anovulation.

Participants with irregular menstrual cycles were followed up with ovulation assessment in three consecutive menstrual cycles. If ovulation was evident in all three cycles they were termed as having regular ovulation while if ovulation was not evident in any of the cycles they were termed anovulatory. Others with ovulation in some cycles were termed as having inconsistent ovulation. All participants with either anovulation or inconsistent ovulation were grouped together as having ovulatory dysfunction.

The study recruited 518 females who attended the clinic during the study period from January 2008 to April
2011. Clinical assessment was carried out in all of them and the assessment of ovulation was carried out in 411 . The tubal patency tests were undertaken in 196 participants. Approval for the study was obtained from the Ethics Review Committee of the Faculty of Medicine, University of Kelaniya.

\section{Results}

Of the 518 couples recruited, $73 \%(n=378)$ had primary infertility while the remaining $27 \%(\mathrm{n}=140)$ had secondary infertility. The mean (SD) age of the female partner was 29.3 (4.9) years while it was 32.5 (5.3) years in the male partner. The mean (SD) duration of infertility was 35 (27) months with a range of 12-144 months.

Among the females $88.8 \%(n=460)$ were vaccinated against rubella and one participant had a history of the infection while $6.2 \%(n=32)$ were unaware of their immune status and $4.8 \%(n=25)$ were not vaccinated.

Of the total study population of 518 couples, assessment of ovulation was carried out in 411 (79.3\%). The 107 females who were excluded from the analysis included 16 on medication for pre-existing endocrinopathies, 16 with previous ovarian surgery, 14 women with partners having severe male factor infertility and 61 who were lost to follow up.

Regular ovulation was observed in $47.0 \%(n=193)$ of the sample. Those with ovulatory dysfunction included $38 \%(n=157)$ with anovulation and $15 \%(n=61)$ with inconsistent ovulation. The association between clinical features and investigatory findings with ovulatory dysfunction is shown in Table 1. The distribution of participant with ovulatory dysfunction according to WHO classification and the association with clinical observations are shown in Table 2.

Transvaginal ultrasound scanning demonstrated uterine fibroids in $5.21 \%(n=27)$. However, the uterine cavity was distorted in three of them only. Myometrial appearance suggestive of adenomyosis was noted in three while an endometrial polyp was diagnosed in two. One woman was found to have a uterine septum, which was confirmed by diagnostic hysteroscopy. Tubal patency was assessed in $37.8 \%(n=196)$. This included 139 women who underwent HSG and 57 who underwent diagnostic laparoscopy with chromotubation. Bilateral tubal patency was confirmed in $91.3 \%(n=176)$, while tubal occlusion was unilateral in $9.1 \%(n=18)$ and bilateral in $1 \%(n=2)$.

Abnormalities in sexual function were noted in $10.8 \%$ $(n=56)$ couples studied. Commonest abnormality was irregular coitus seen among 6.9\% $(\mathrm{n}=36)$ followed by infrequent coitus $(2.8 \%, \mathrm{n}=15)$, erectile dysfunction $(0.9 \%$, $\mathrm{n}=5)$ and abnormalities in ejaculation $(0.7 \%, \mathrm{n}=4)$. 
Table 1. The association between clinical features and investigatory findings of the study participants and ovulatory dysfunction

\begin{tabular}{|c|c|c|c|}
\hline Clinical feature & $\begin{array}{l}\text { Ovulatory dysfunction } \\
\qquad n=218\end{array}$ & $\begin{array}{c}\text { Regular ovulation } \\
n=193\end{array}$ & $\begin{array}{l}\text { Association and } \\
\text { significance }\end{array}$ \\
\hline \multicolumn{4}{|l|}{ Age of the subject } \\
\hline Less than $30 \mathrm{yrs}$ & 141 & 87 & \\
\hline vs. $30-35$ yrs $(n=115)$ & 51 & 64 & OR $0.49(0.31-0.77)$ \\
\hline vs. $>35$ yrs $(n=68)$ & 26 & 42 & OR $0.38(0.22-0.66)$ \\
\hline \multicolumn{4}{|l|}{ Menstrual abnormalities } \\
\hline Regular cycles $(\mathrm{n}=250)$ & 62 & 188 & \\
\hline vs. irreg. cycles (n=57) & 52 & 7 & OR 31.5 (12.1-81.8) \\
\hline vs. cycles of $>90 d(n=104)$ & 104 & $\mathbf{0}$ & OR 630.4 (39-10159) \\
\hline \multicolumn{4}{|l|}{ Body habitus } \\
\hline Normal BMI (n=195) & 95 & 100 & \\
\hline vs. overweight $(n=130)$ & 80 & 50 & OR 1.68 (1.07-2.64) \\
\hline vs. obese $(n=39)$ & 29 & 10 & OR 3.05 (1.42-6.55) \\
\hline Goitre $(\mathrm{n}=27)$ & 11 & 16 & OR $0.59(0.27-1.28)$ \\
\hline Acanthosis nigricans $(n=40)$ & 35 & 5 & OR 7.19 (2.79-18.54) \\
\hline Hirsutism (n=78) & 64 & 14 & OR 5.31 (2.88-9.81) \\
\hline Polycystic ovary synd. (n=130) & 127 & 3 & OR 88.3 (27.5-284) \\
\hline \multicolumn{4}{|l|}{ Day 2 FSH level } \\
\hline vs. normal FSH (n=396) & 210 & 186 & \\
\hline vs. FSH > 20IU/L (n=14) & 7 & 7 & OR $0.89(0.37-2.10)$ \\
\hline LH:FSH > $1(n=102)$ & 78 & 24 & OR 3.92 (2.36-6.52) \\
\hline \multicolumn{4}{|l|}{ TSH level } \\
\hline Normal TSH level $(\mathrm{n}=361)$ & 19 & 342 & \\
\hline vs. low TSH level & 2 & 0 & \\
\hline vs. high TSH level (n=48) & 32 & 16 & OR $1.92(1.03-3.61)$ \\
\hline High prolactin levels ( $\mathrm{n}=39$ ) & 26 & 13 & OR $1.87(0.94-3.73)$ \\
\hline High testosterone levels $(n=18)$ & 16 & 02 & OR 7.56 (1.79-32.0) \\
\hline
\end{tabular}

Expressed as number and odds ratio with $95 \%$ CI. Statistically significant associations in bold. 
Table 2. The distribution of subjects with ovulatory dysfunction according to WHO classification and the association with clinical observations $(n=193)$

\begin{tabular}{lc}
\hline The underlying causes studied & No (\%) \\
\hline WHO group I (hypogonadotropic hypogonadism) $(\mathrm{n}=1)$ & \\
Low FSH levels $(<2 \mathrm{IU} / \mathrm{L})$ & $1(0.4 \%)$ \\
WHO group II (normogonadotropic hypogonadism) $(\mathrm{n}=188)$ & \\
Low BMI $\left(<18.5 \mathrm{~kg} / \mathrm{m}^{2}\right)$ & $16(7.3 \%)$ \\
Obesity $(>30 \mathrm{~kg} / \mathrm{m} 2)$ & $29(13.3 \%)$ \\
Polycystic ovary syndrome & $132(60.5 \%)$ \\
High FSH $(>20 \mathrm{IU} / \mathrm{L})$ with normal oestradiol & $7(3.2 \%)$ \\
Low TSH levels (hyperthyroidism) & $2(0.9 \%)$ \\
High TSH levels (hypothyrodism) & $32(14.6 \%)$ \\
Raised prolactin levels & $26(11.9 \%)$ \\
WHO group III (hypergonadotropic hypergonadism) $(\mathrm{n}=4)$ & \\
Premature ovarian failure & $4(1.8 \%)$ \\
\hline
\end{tabular}

\section{Discussion}

Infertility is a widely expanding specialty with much research being carried out in different settings. Most studies carried out in Sri Lanka on infertility have focused on male factor infertility $[10,11]$. This study was aimed at evaluating the aetiological factors in female infertility. The large sample size and the non-contrived study population improved the external validity of the study.

This study demonstrated that over $4 \%$ were not vaccinated against rubella while another $6 \%$ was not aware of their vaccination status. This reiterates the relevance of assessing rubella immune status in peri-conceptional care.

This study shows that ovulatory dysfunction is evident in over half of the patient population. Previous studies have demonstrated much smaller proportions (20$30 \%)$ of ovulatory dysfunction $[1,7,12]$. A possible explanation for this higher rate in our study is the follow up we employed in assessment of ovulation. In a study where such follow up is not used, those with inconsistent ovulation will be erroneously classified as ovulatory if they ovulated in the index cycle. Nearly $16 \%$ of the population studied had such inconsistent ovulation. Therefore, this study demonstrates the importance of follow up in assessing ovulation in all women who lack evidence of regular ovulation such as regular menstrual cycles.

WHO group II ovulatory dysfunction was noted in over $97 \%$ of the subjects while polycystic ovary syndrome was the most common clinical entity seen in over $60 \%$ of subjects with ovulatory dysfunction. Hormone abnormalities associated with ovulatory dysfunction included a reversed LH:FSH ratio, a high TSH level and a raised testosterone level. However, it should be emphasised that all these are changes commonly seen in PCOS, rather than being endocrinopathies in isolation.

Uterine and pelvic pathologies were uncommon and previous studies have demonstrated similar findings [13]. Occlusion of either one or both tubes was noted in nearly $10 \%$. Since such investigations were carried out only in a subset of women in whom it was clinically indicated, it was prone to a selection bias leading to an over-estimation. Bilateral tubal occlusion leading to absolute infertility was detected only in $1 \%$ of the study population. This justifies the clinical protocols used by us as well as many other infertility centres of carrying out such investigations only in the presence of long standing infertility or prior to interventions such as intrauterine insemination (IUI).

An interesting area studied was the assessment of sexual function among couples. This is an area often neglected in both research as well in clinical management of infertility. Abnormalities in sexual function were seen in over $10 \%$ of our study population. The most common among these were irregular coitus (absence of regular coitus in the preceding month) followed by infrequent coitus (a coital frequency of less than once a week). In many instances this was the result of temporary absence of the partner due to demands of occupation rather than lack of sexual desire. The other causes of sexual dysfunction such as abnormalities in erection and ejaculation were uncommon and affected less than $2 \%$ of the population. This study highlights the importance of 
assessing sexual function of the couples that present for infertility treatment.

In conclusion, this study demonstrates that ovulatory dysfunction and abnormalities in sexual function are common findings in an infertile population. The proportion of women with pelvic pathology and bilateral tubal occlusion were low and investigations for such abnormalities should not be included in the initial assessment but should be reserved for a subset of women at an increased risk or those undergoing interventions such as IUI to improve the cost effectiveness.

\section{Acknowledgements}

This study was funded through research grants from the National Science Foundation of Sri Lanka (Grant No RG/2007/HS/08) and the National Research Council (Grant no 06-30).

\section{References}

1. Evers J. Female subfertility. The Lancet 2002; 360: 151-9

2. Balen AH, Rutherford AJ. Management of infertility. British Medical Journal 2007; 335: 608-11.

3. Iammarrone E, Balet R, Lower AM, Gillott C, Grudzinskas JG. Male infertility. Best Practice and Research in Clinical Obstetrics and Gynaecology 2003; 17: 211-29.

4. ESHRE Capri Workshop Group. Diagnosis and management of the infertile couple: missing information. Human Reproduction Update 2004; 10: 295-307.
5. Hull MGR, Glazeber CMA, Kelly NJ, et al. Population study of causes, treatment and outcome of infertility. British Medical Journal 1985; 291: 1693-7.

6. Hart R. Unexplained infertility, endometriosis, and fibroids. British Medical Journal 2003; 327: 721-4.

7. Adamson GD, Baker VL. Subfertility: causes, treatment and outcome. Best Practice and Research Clinical Obstetrics and Gynaecology 2003; 17: 169-85.

8. World Health Organization. The epidemiology of infertility: Report of a WHO scientific group. Geneva: World Health Organization 1975.

9. National Institute for Health and Clinical Excellence. Investigation of fertility problems and management strategies. Fertility: Assessment and Treatment for People With Fertility Problems. London, RCOG Press 2004: 39-51.

10 Fernando DMS. The prevalence of antisperm antibodies, their effects on infertile couples and a possible treatment intervention for autoimmune infertility (Thesis). Colombo: University of Colombo 2001.

11 Wijeratne S, Seneviratne HR, Ratnasooriya WD. Reproductive hormones in seminal plasma; its effect on semen quality. The Ceylon Journal of Medical Science 2005; 48: 53-60.

12 Hamilton-Fairley D, Taylor A. ABC of subfertility; Anovulation. British Medical Journal 2003; 327: 546-9.

13 Bayasgalan G, Naranbat D, Tsedmaa B, Tsogmaa B, Sukhee $\mathrm{D}$, et al. Clinical patterns and major causes of infertility in Mongolia. Journal of Obstetrics and Gynaecology Research 2004; 30: 386-93. 